\title{
Intellectual disability-developmental delay-contractures syndrome
}

INSERM

\section{Source}

INSERM. (1999). Orphanet: an online rare disease and orphan drug data base. Intellectual disability-developmental delay-contractures syndrome. ORPHA:3454

Intellectual disability-developmental delay-contractures syndrome, formerly known as Wieacker-Wolff syndrome, is a severe X-linked recessive neurodevelopmental disorder characterized by severe contractures (arthrogryposis; see this term) and intellectual disability. 